\title{
A comparative study of finger vein recognition by using Learning Vector Quantization
}

\author{
Hardika Khusnuliawati ${ }^{1}$, Chastine Fatichah ${ }^{1}$, and Rully Soelaiman ${ }^{1}$
}

\begin{abstract}
This paper presents a comparative study of finger vein recognition using various features with Learning Vector Quantization (LVQ) as a classification method. For the purpose of this study, two main features are employed: Scale Invariant Feature Transform (SIFT) and Local Extensive Binary Pattern (LEBP). The other features that formed LEBP features: Local Multilayer Binary Pattern (LmBP) and Local Directional Binary Pattern (LdBP) are also employed. The type of images are also become the base of comparison. The SIFT features will be extracted from two types of images which are grayscale and binary images. The feature that have been extracted become the input for recognition stage. In recognition stage, $L V Q$ classifier is used. $L V Q$ will classify the images into two class which are the recognizable images and non recognizable images. The accuracy, false positive rate (FPR), and true positive rate (TPR) value are used to evaluate the performance of finger vein recognition. The performance result of finger vein recognition becomes the main study for comparison stage. From the experiments result, it can be found which feature is the best for finger vein reconition using $L V Q$. The performance of finger vein recognition that use SIFT feature from binary images give a slightly better result than uisng LmBP, LdBP, or LEBP feature. The accuracy value could achieve 97,45\%, TPR at 0,9000 and FPR at 0,0129.
\end{abstract}

Keywords-finger vein recognition, SIFT, LEBP, LVQ

\section{INTRODUCTION}

The finger-vein pattern is one of biometric areas that can be used for personal identification. In general, finger-vein recognition consists four stages: image capturing, preprocessing, feature extraction and recognition. A feature extraction stage gains a lot of attention from many researchers. The examples of feature extraction methods which already used for finger-vein recognition are line tracking [1], maximum curvature [2], and mean curvature [3]. Line tracking extracts the finger-vein pattern from the unclear image by using line tracking that starts from various positions. Maximum curvature developed a method of calculating local maximum curvatures in crosssectional profiles of a vein image. Whereas mean curvature views the vein image as a geometric shape and finds the valley-like structures with negative mean curvatures. However, those methods that already mentioned have not yet been focused on extracting fitur from degraded images. The features extracted from degraded images can not become an optimal features. That condition will affect the performance result of finger vein recognition. It becomes important to choose the features that resistant to degrading conditions.

Image degradation can be caused by scaling, rotation effect, translation effect, and varying illumination conditions. Those conditions may affect the accuracy of recognition stage and reduce the performance of system.

${ }^{1}$ Hardika Khusnuliawati, Chastine Fatichah, and Rully Soelaiman are Departement of Informatics Engineering, Institut Teknologi Sepuluh
One of the features which has been widely used for finger-vein recognition with degraded images is Scale Invariant Feature Transform (SIFT). As a mentioned in [4], SIFT has a capability to extract feature from images that degraded by scaling, rotation effect, and translation effect. The implementation of SIFT feature can be found in several cases such as object recognition [4], image retrieval [5], and biometrics [6]. For object recognition case in [4], matching algorithm is used to recognize object based on SIFT feature. Codebook is generated from SIFT feature as one of the feature that used in image retrieval case [5]. SIFT feature becomes one of features that clustered using K-Means algorithm to generate codebook. SIFT feature also already used in biometrics area, as example in palm vein recognition [6]. From that paper, it can be found that SIFT feature gives a satisfaction performance result. SIFT feature can be extracted from grayscale images or binary images.

LEBP which introduced by Liu (2014) is the other feature that affords to extract feature from degraded images [5]. LEBP feature is the development of LBP feature [8]. It can resist to extract feature from image with variations in illumination conditions. The robustness can be achieved since the operator is by definition invariant against any monotonic transformation of the gray scale. SIFT and LEBP feature have potential to be a afeature that used in recognition stage for degraded images.

In recognition stage, there are two types of methods that can be used. The first one is using threshold distance [7] and the other one is using classification methods. If we use the threshold distance, we will utilize one of the distance calculation methods. Euclidean distance and hamming distance are the common calculation distance method that has been used. There is a weakness when we use threshold distance. The accuracy result from finger vein recognition will be strongly depend on the threshold value. It can make the optimal accuracy result hard to find. So that, classifictaion method is implemented for a number of studies in many biometric cases, such as SVM [9] and LVQ [10]. In [9], SVM is combined with gabor filter as a feature in finger vein recognition system. Menawhile in [10], gabor filter feature is used as an input for LVQ classifier to recognize a facial expression along with other feature like LBP, LDP, LDA, and PCA. LVQ also has been commonly used in biometric areas, for example in face detection [11]. For face detection, PCA feature is uesd. But, there is currently no implementation of LVQ in finger-vein recognition using the potential feature that has been mentioned above.

In this paper, we will perform a detailed comparative study for finger-vein recognition using SIFT feature and LEBP feature with LVQ method as classifier. In a

Nopember, Surabaya, 60111, Indonesia. E-mail hardika.khusnulia@gmail.com; chastine@cs.its.ac.id; rully@if.its.ac.id. 
systematic manner, we will compare and evaluate the results obtained based on each technique and present the benefits associated with each paradigm.

\section{METHOD}

Finger-vein recognition which explained in this paper consists of four main stages: prerocessing, feature extraction, recognition, and comparative study. SIFT and LEBP are features that extracted from images and will be analysed. LVQ method wil be used in recognition stage. A flowchart of finger-vein recognition system is provided in Figure 1.

\section{A. Preprocessing}

The preprocessing steps that applied for degraded images are selecting ROI, size normalization, and image enhancement. The methods that are used for image enhancement are median filter, frangi filter, CLAHE, and morphology operation. Median filter [12] is used to remove noise from images which follows equation(2).

$\hat{\theta}_{\text {med }}=\left\{\begin{aligned} \frac{1}{2}\left(X_{(n / 2)}+X_{(1+n / 2)}\right), & \text { for even } n \\ X_{(n+1 / 2)}, & \text { for odd } n\end{aligned}\right.$

where $X_{(i)}$ is the ith image from the n number of images. To get a clear pattern of finger vein from images, CLAHE [13] and frangi filter [14] are applied. CLAHE can enhance the contrast of image which follows the equation(3)

$$
i_{e n h(i)}=\frac{c d f(i)-c d f_{\min }}{\left(w_{x} \times w_{y}\right)-c d f_{\min }} \times(L-1)
$$

where $i_{\text {enh }}$ is the new value of intensity of image with the window size $w_{x} \times w_{y}, c d f(i)$ is cumulative density function of intensity $i, c d f_{\min }$ is the minimum value of cumulative density function from the whole image, and $L$ is the length of grey level value. Meanwhile, frangi filter is examined with the purpose of developing a vessel enhancement filter. A vesselness measure is obtained on the basis of all eigenvalues of the Hessian. Hessian Matrix can be obtained from the calculation of derivative Gaussian function. The equation which show the Hessian Matrix is followed by equation(4).

$$
\begin{aligned}
\mathcal{H}_{\sigma}(I, x) & =\frac{\partial^{2} I_{\sigma}}{\partial x^{2}} \\
& =I(x) * \frac{\partial^{2} \mathcal{G}_{\sigma}(x)}{\partial x^{2}}
\end{aligned}
$$

where $\mathrm{x}$ is random point from image $\mathrm{I}$ and $\mathcal{G}_{\sigma}$ is a Gaussian function standard deviation $\sigma$.

Then, morphology method is apllied to images, such as dilation, erotion, closing, and opening. Morphology method [15] use structuring elements as probes to analyze the image. In dilation operation, the objects in the binary image will expand so the territory becomes wider. In the other hand, in erotion operation, the objects in the binary image will be narrowed. If the image $\mathrm{A}$ is processed using structuring element $B$ where $\beta$ as a member of $\mathrm{B}$, the notation for dilation and erosion are shown respectively in equation(4) and equation(5).
$A \oplus B=\cup_{\beta \epsilon B} A+\beta$

$A \ominus B=\bigcap_{\beta \epsilon B} A-\beta$

Dilation and erotion can also be combined forming the other morphological operations, closing and opening. At the opening operation, the image A will be processed by erosion operation, the followed by dilation operation. Meanwhile, at the closing operation, the image A will be processed by dilation operation, the followed by erotion operation. The equation for opening and closing operation are shown respectively in equation(6) and equation(7).

$A^{\circ} B=(A \ominus B) \oplus B$

$A \cdot B=(A \oplus B) \ominus B$

The image results of CLAHE and Frangi Filter can be seen in Figure 2. Meanwhile, the image results of median filter and morphological operations can be seen in Figure 3. The image results from those method are grayscale and binary images with a clear vein pattern. Finally, The binary and grayscale images resize in sizes 250x95 pixels. The example of the images can be seen in Figure 4.

\section{B. Feature Extraction}

There are two features that will be obtained from images which produced from preprocessing stage. They are SIFT feature and LEBP feature.

\section{1) SIFT Feature}

SIFT feature is obtained from grayscale images and binary images. The steps to get this feature follow the steps that explained in [4]. There are four main steps which must be followed to get SIFT descriptor. The first step is scale-space extrema detection. Difference-of Gaussian (DoG) method is used to get scale-space extrema which follows equation(8).

$$
\begin{aligned}
D(x, y, \sigma) & =(G(x, y, k \sigma)-G(x, y, \sigma)) \cdot I(x, y) \\
& =L(x, y, k \sigma)-L(x, y, \sigma)
\end{aligned}
$$

where $G(x, y, \sigma)$ is Gaussian scale variable, $I(x, y)$ is the image, $\sigma$ is scale factor and $\mathrm{L}(\mathrm{x}, \mathrm{y}, \mathrm{k} \sigma)$ is the scale-space. From the first step, we will get local extrema as keypoints candidate. The next step is eliminating the keypoints candidate which have low contrast and possibility of being noise, and located around the edges. Hessian matrix is used to determine which keypoint candidate will be eliminated. After the stabil keypoint obtained, the next step is determining the gradient $m(x, y)$ and the orientation $\theta(x, y)$ of the stable keypoints which follow respectively in equation(9) and equation(10).

$$
\begin{aligned}
& m(x, y)=\sqrt{\begin{array}{c}
(L(x+1, y)-L(x-1, y))^{2} \\
+(L(x, y+1)-L(x, y-1))^{2}
\end{array}} \\
& \theta(x, y)=\tan ^{-1}\left(\frac{L(x+1, y)-L(x-1, y)}{(L(x, y+1)-L(x, y-1))}\right)
\end{aligned}
$$

The last step is constructing the descriptor of SIFT feature. Every keypoint will be represented in 128 vector length. So that, the final results of SIFT feature extraction are list of keypoints with the descriptor value. 
To make SIFT feature becomes available to be input for LVQ classifier, SIFT descriptors is transformed into histogram representation using Bag Of Visual Words (BVW) [16]. This algorithm agglomerates the list of keypoints from training data using K-Means algorithm to get the vocabulary. Then, the list of keypoints from testing data will be mapped into the vocabulary.

\section{2) LEBP Feature}

LEBP features are obtained from grayscale images using vein pattern as a mask. LEBP features are the result of combination process between LmBP feature and LdBP feature which represented in 72 binaries vector [7]. LmBP feature is extended version of LBP feature. If LBP feature use $3 \times 3$ neigbouring pixels, LmBP feature will use $7 \times 7$ neighbouring pixels. There are three layer surrounds the center pixel. LmBP feature represents in 64 binaries vector.

There are two comparison process that must be followed to get LmBP feature. First, the comparison between the center pixel of kernel with the neighbouring pixels. The result of the first comparison is 48 binaries feature vector. Second, the comparison between pixels in the inner layer and pixels in the outer layer. It will produce a feature vector in 16 binaries length. The binary value $b_{i}$ of vector feature can be obtained by following equation(11).

$b_{i}=\left\{\begin{array}{c}0, \text { if } p<q \\ 1, \text { otherwise }\end{array}\right.$

where $p$ is pixel in the center of kernel or in the inner layer and $\mathrm{q}$ is neighbouring pixel or in the outer layer.

LdBP feature is the modified version of Local Directional Code (LDC). The size of kernel that used to get LdBP feature is $3 \times 3$. The first step that must be followed to get LdBP feature is calculating LDC value. It uses the difference of the neighbours of a pixel as the two components $\mathrm{v}_{\mathrm{v}}$ and $\mathrm{v}_{\mathrm{h}}$. It is followed equation(12) and equation(13) to obtain $v_{v}$ and $v_{h}$ value respectively.

$v_{h}=a_{7}-a_{3}$

$v_{v}=a_{5}-a_{1}$

where $a_{7}$ is the neighbouring pixel which appropriately located in the left of the center pixel and $a_{3}$ is the neighbouring pixel which appropriately located in the right of the center pixel. Meanwhile $\mathrm{a}_{5}$ is the neighbouring pixel which appropriately located in the bottom of the center pixel and $a_{1}$ is the neighbouring pixel which appropriately located above of the center pixel.

The vector of LdBP feature is the result of the comparison value between the $t$ value of the central pixel with the $t$ value of neighbouring pixels, where the $t$ value is obtained by following equation(14) with the $T$ value is the number of neighboring pixels that are involved and the value of $\theta^{\prime}$ obtained by following equation(15). While the value of the gradient orientation $\theta$ obtained by following equation equation(16).

$t=\bmod \left(\frac{\theta^{\prime}}{2 \pi / T}, T\right)$

$\theta^{\prime}=\arctan 2\left(v_{v}, v_{h}\right)+\pi, \arctan 2\left(v_{v}, v_{h}\right)$

$$
=\left\{\begin{array}{ll}
\theta & v_{h}>0, v_{v}>0 \\
\pi+\theta & v_{h}>0, v_{v}<0 \\
\theta-\pi & v_{h}<0, v_{v}<0 \\
\theta & v_{h}<0, v_{v}>0
\end{array}, \theta \in\left[-\frac{\pi}{2}, \frac{\pi}{2}\right]\right.
$$

$\theta(m)=\arctan \left(\frac{v_{h}}{v_{v}}\right)$

The result of the comparison between $t$ values is 8 binaries feature vector. If the $t$ value of center pixel is greater than the $t$ value of neighbouring value, th ebinary value will be signed 1 , otherwise it will be 0 .

Every eight bits of LEBP vector converts into decimal values with a range between 0 to 255 . The decimal values that obtained are mapped into histogram representation.

\section{LVQ Classifier}

LVQ is supevised learning that closely related with Vector Quantization (VQ) and Self Organizing Map (SOM) [11]. This method is built by Kohonen. While VQ and the basic SOM are unsupervised clustering and learning methods, LVQ describes supervised learning. On the other hand, unlike in SOM, no neighborhoods around the winner node are defined during learning in the basic LVQ, whereby also no spatial order of the codebook vectors is expected to ensue.

LVQ algorithm is divided into three types, namely LVQ1, LVQ-2, and LVQ-3. The LVQ1 and the LVQ3 define a more robust process. For the LVQ1 the learning rate can approximately be optimized for quick convergence. In this study, we used LVQ-1 that consist of two layers. They are competitive layer or also called sub-classes and output layer. The architecture of classification system using LVQ can be seen in Figure 5.

The competitive layer learns to classify input vectors, meanwhile the output layer transforms the competitive layer's classes into target classifications. In every competitive layer, there is initialization process of a weighting vector that has similarity to the input vector. Each neuron in the competitive layer will be found the best matches to winning neuron. Distances between input and neurons are calculated from their positions with a ecludiean distance function $D(a, b)$ which follow equation(17). The value of $b_{i}$ is representing input vector and $a_{i}$ represent the weight value of neurons in the competitive layer. The larger the hidden layer the more clusters the competitive layer can learn, and the more complex mapping of input to target classes can be made.

$D(a, b)=\sqrt{\sum_{i=1}^{n}\left(b_{i}-a_{i}\right)^{2}}$

Then the class in the linear layer neuron which associated with winning neuron is set into a high value. The linear layer merges groups of the competitive layer clusters into the classes defined by the target data. The competitive layer neuron that is the closest to the input is determined and the corresponding output neuron is called the winner neuron. The weights of the connections to this neuron are then updated. The weighting vector is updated by following equation(18).

$w_{i}(n)=\left\{\begin{array}{lr}w_{i}(n-1)+\alpha\left(p-w_{i}(n-1)\right) \text { if the result is true } \\ w_{i}(n-1)-\alpha\left(p-w_{i}(n-1)\right) & \text { otherwise }\end{array}\right.$ 
where $w_{i}(n)$ is the new weight after $n^{\text {th }}$ iteration, $\alpha$ is the learning rate value, and $\mathrm{p}$ is the input vector.

D. Comparative Study

The results from feature extraction will be the input for LVQ classifier. The features that are used in this study are SIFT, LmBP, LdBP, and LEBP feature. All of the features are represented in histogram vector.

To see the performance of the finger vein recognition system, the confusion matrix that can be seen in Table 1 is performed. True Positive (TP) means a case in which classifier predicted true consorts with the actual condition. Meanwhile, False Positive (FP) means a case in which classifier predicted true but it is false in actual condition. The other term is True Negative (TN) which means a case in which classifier predicted false consorts with the actual condition. And the last term is False Negative (FN) which means a case in which classifier predicted false but it is true in actual condition.

The value of accuracy, FPR and TPR is used as a measurement of system performance. This value is derived from the analysis of the confusion matrix. From the results of the analysis, the equation to calculate the accuracy, FPR, and TPR shown in equation(2), equation(3), and equation(4) respectively.

$$
\begin{array}{ll}
\text { accuracy } & =(T P+T N) /(T P+F N+F P+T N) \\
T P R & =T P /(T P+F N) \\
F P R & =F P /(F P+T N)
\end{array}
$$

The accuracy, TPR, and FPR value from LVQ classifier will be the materials that analyzed. The highest value of accuracy and TPR along with the lowest value of FPR value indicates the best combination between feature and LVQ classifier.

\section{RESULTS AND DISCUSSION}

The comparative experiments between SIFT and LEBP using LVQ classifier were conducted on the finger-vein dataset from Hong Kong Polytechnic University [10] that use finger vein images from 50 persons.

There are five experiments which have been done. The first and second experiment use combination of SIFT feature and LVQ classifier. The difference between the first and second experiment is the images in which the feature extracted. For the first experiment, SIFT feature is extracted from binary images. Meanwhile for the second experiment, SIFT feature is extracted from grayscale image.

The remaining experiments will use $\mathrm{LmBP}$, LdBP, and LEBP feature to be combined with LVQ classifier. All of the experiments must be set into the same condition. The condition of finger vein recognition using LVQ depends on influential parameters which are learning rate, number of hidden layers, and number of epochs. For all of the experiments, the learning rate value set with into 0,01 . Meanwhile the number of hidden layer set into 300 and the number of epochs set into 500 .

Table 2 shows the accuracy, FPR, and TPR value from the use of SIFT, LmBP, LdBP, and LEBP feature in the finger vein recognition using LVQ. The average accuracy that can be achieved is $97 \%$. It also can be seen if the accuracy value with SIFT feature can achieved a better result than using the other features. The highest accuracy is $97,45 \%$ which obtained by using SIFT feature from binary images then followed by the accuracy that obtained from grayscale images with value $97,34 \%$. The least accuracy is obtained by using LdBP feature with value 96,38\%. Meanwhile, by using LmBP and LEBP feature, the accuracy can be achieved $97,24 \%$ and $97,32 \%$ respectively.

The average FPR that can be achieved is 0,0145 . It also can be seen if the FPR value with SIFT feature can achieved a better result than using the other features. The lowest FPR value is 0,0129 which obtained by using SIFT feature from binary images then followed by the accuracy that obtained from grayscale images with value 0,0135 . The highest FPR value is obtained by using LdBP feature with value 0,0184 Meanwhile, by using LmBP and LEBP feature, the accuracy can be achieved 0,0141 and 0,0137 respectively.

The average TPR that can be achieved is 0,7000 . It also can be seen if the TPR value with SIFT feature can achieved a better result than using the other features. The highest TPR value is 0,9000 which obtained by using SIFT feature from binary images then followed by the accuracy that obtained using LEBP feature with value 0,8300 . The lowest TPR value is obtained by using LdBP feature with value 0,2350 . Meanwhile, by using $\mathrm{LmBP}$ and SIFT feature from grayscale images, the accuracy can be achieved 0,7800 and 0,8200 respectively.

From the accuracy result, it can be said that SIFT feature from binary images give a slightly better result than LmBP,LdBP, and LEBP feature with a different of $0,1 \%$. The FPR value which obtained by using SIFT feature also give the best result. The most significant result can be seen from the TPR result which show SIFT feature from binary result give the higher value than $\mathrm{LmBP}, \mathrm{LdBP}$, and LEBP.

Eventhough using SIFT feature from binary images gives the best result, the other feature LmBP, LdBP, and LEBP are also effective to implement on degraded images and to be combined with LVQ classifier.

\section{CONCLUSION}

This paper compares the accuracy of finger vein recognition using SIFT and LEBP features with LVQ method. To compare both features, SIFT feature and LEBP feature are modified into the same representation that is histogram vector. The conditions of LVQ classifier are also set to the same. So that, the fair comparisons can be achieved. From the accuracy results, SIFT feature from binary images give a slightly better result than LmBP, LdBP, and LEBP but not really significant. The most significant result can be seen from FPR and TPR value which also shown SIFT feature from binary images is the best choice to combine with LVQ classifier.

For the future research, the possibility of integration between features can be done. Because feature SIFT and LEBP have the different capability to compromise with the caused of degraded images, it can produced a better result if we use the information that obtained from both features. 


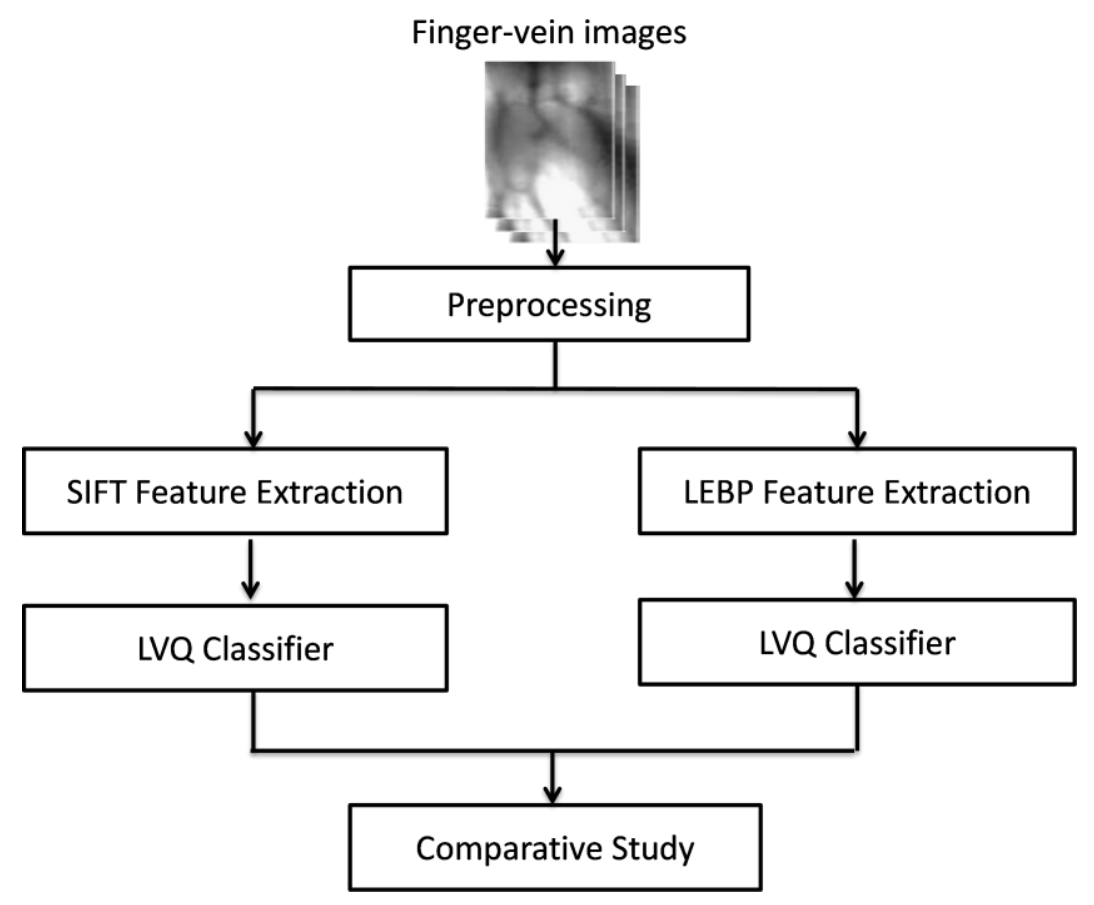

Figure. 1. Flowchart of finger-vein recognition system

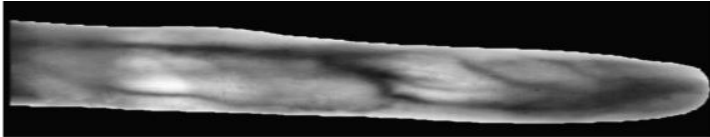

( a )

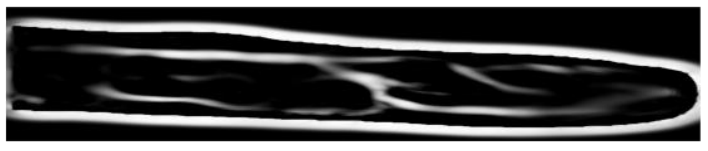

( c )

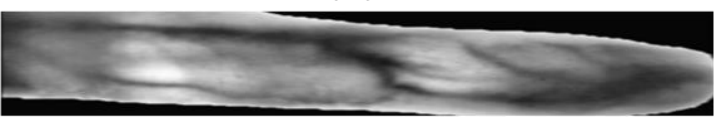

( e )

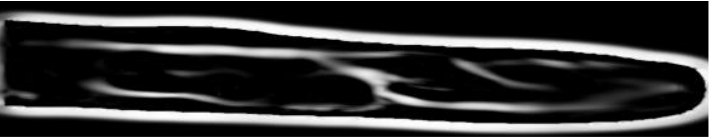

( b )

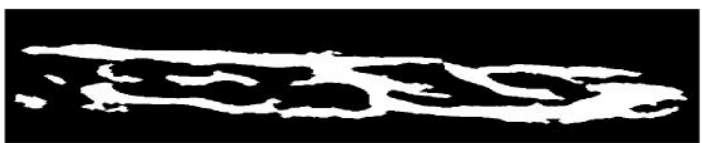

(d)

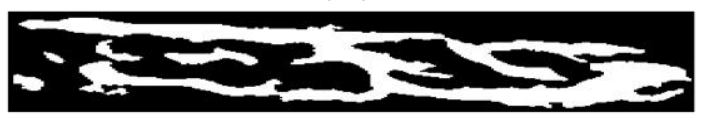

( f)

Figure. 2. The image of the preprocessing stage (a) the image of the CLAHE (b) the image of the Frangi Filter 
The $2^{\text {nd }}$ International Seminar on Science and Technology

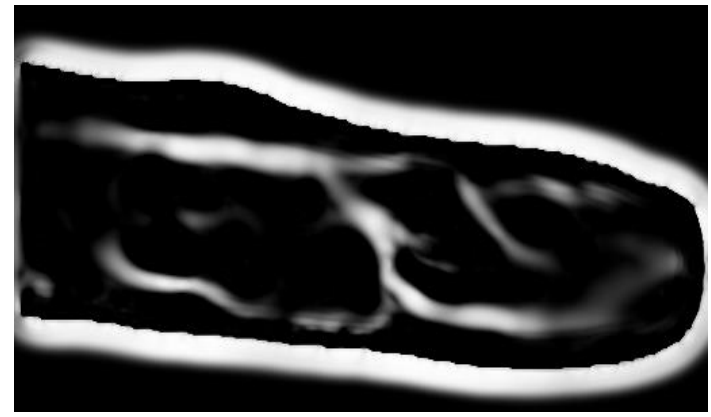

(a)

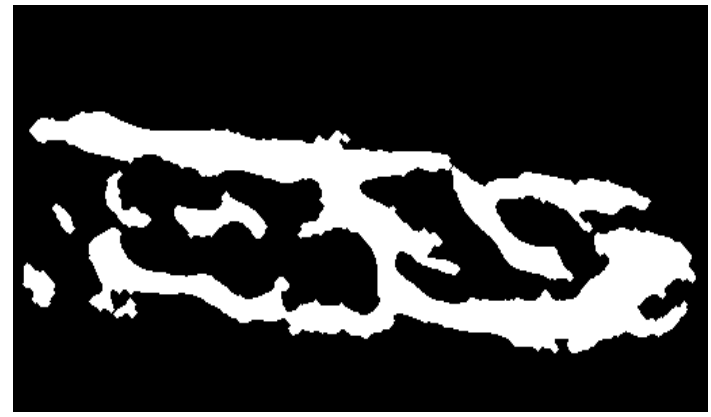

(b)

Figure. 3. The image of the preprocessing stage (a) the image of the Median Filter (b) the image of the Morphology Operations

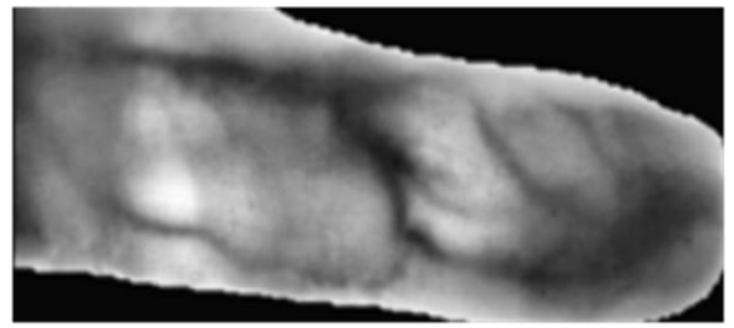

(a)

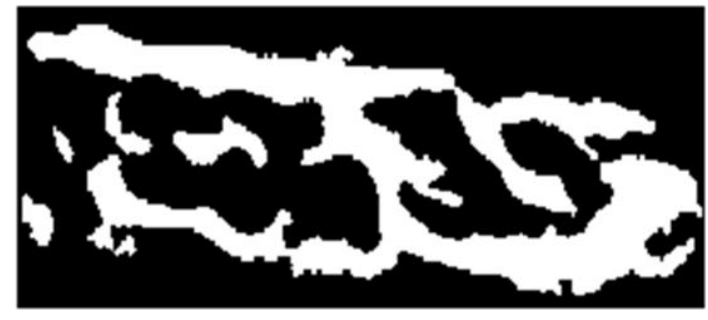

(b)

Figure. 4. The images result of cropping and image size normalization stage (a) The grayscale image result from CLAHE (b) The binary image morphological operations and normalizing the image size

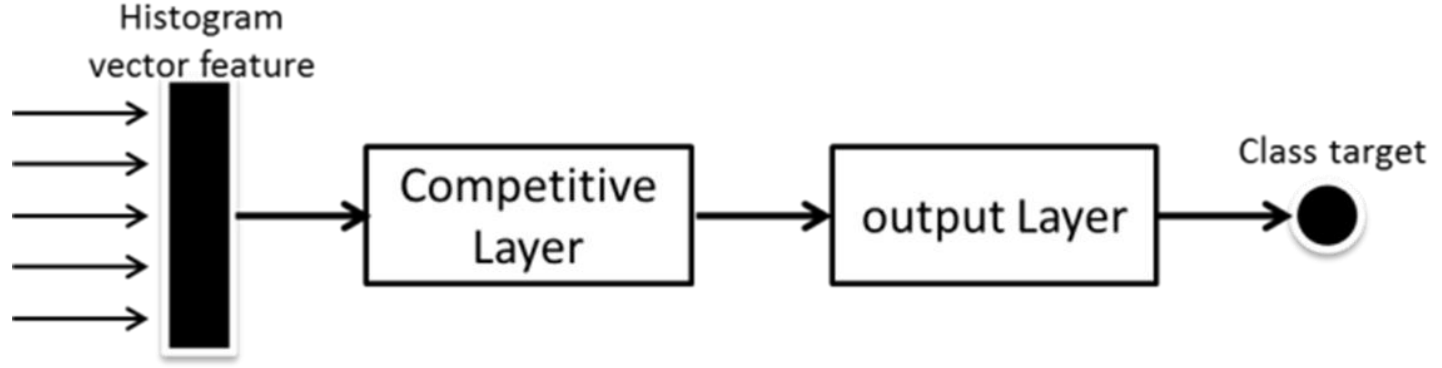

Figure. 5. Architecture of finger-vein recognition system using LVQ 
TABLE 1.

CONFUSSION MATRIX

\begin{tabular}{ccc}
\hline \multirow{2}{*}{ Prediction } & \multicolumn{2}{c}{ Actual } \\
\cline { 2 - 3 } & Within Class & Between Class \\
\hline Within Class & $\begin{array}{c}\text { True Positive } \\
\text { (True Accept, Hit) }\end{array}$ & $\begin{array}{c}\text { False Positive } \\
\text { (False Accept, False Alarm) }\end{array}$ \\
\hline \hline
\end{tabular}

TABLE 2.

TABLE OF PERFORMANCES RESULTS

\begin{tabular}{cccc}
\hline \hline Feature & Accuracy & FPR & TPR \\
\hline SIFT (binary) & 97,45 & 0,0129 & 0,9000 \\
SIFT (grayscale ) & 97,34 & 0,0135 & 0,8200 \\
LmBP & 97,24 & 0,0141 & 0,7800 \\
LdBP & 96,38 & 0,0184 & 0,2350 \\
LEBP & 97,32 & 0,0137 & 0,8300 \\
\hline \hline
\end{tabular}

\section{REFERENCES}

[1] N. Miura, A. Nagasaka and T. Miyatake, "Feature extraction of finger-vein patterns based on repeated line tracking and its application to personal identification," Machine Vision and Applications, pp. 194-203, 2004.

[2] N. Miura, A. Nagasaka and T. Miyatake, "Extraction of fingervein patterns using maximum curvature points in image profiles," IEICE TRANSACTIONS on Information and Systems, pp. 11851194, 2007.

[3] W. Song, T. Kim, H. C. Kim, J. H. Choi , H.-J. Kong and S.-R. Lee, "A finger-vein verification system using mean curvature," Pattern Recognition Letters, pp. 1541-1547, 2011.

[4] D. G. Lowe, "Distinctive image features from scale-invariant keypoints.," International journal of computer vision 60.2 , pp. 91-110, 2004

[5] J. Yu, . Z. c. Qin, T. Wan and X. Zhang, "Feature integration analysis of bag-of-features model for image retrieval," Neurocomputing, pp. 355-364, 2013.

[6] P.-O. Ladoux, C. Rosenberger and B. Dorizzi, "Palm vein verification system based on SIFT matching," Advances in Biometrics, pp. 1290-1298, 2009.

[7] F. Liu, G. Yang, Y. Yin and S. Wang, "Singular value decomposition based minutiae matching method for finger vein recognition," Neurocomputing, pp. 75-89, 2014.

[8] T. Ojala, M. Pietikäinen and T. Mäenpää, "Multiresolution grayscale and rotation invariant texture classification with local binary patterns," Pattern Analysis and Machine Intelligence, pp. 971987, 2002.
[9] K. R. Park, "Finger vein recognition by combining global and local features based on SVM," Computing and Informatics 30.2, pp. 295-309, 2012.

[10] G.-J. de Vries, S. Pauws and M. Biehl, "Facial Expression Recognition Using Learning Vector Quantization," Springer International Publishing, pp. 760-771, 2015.

[11] S. Bashyal and G. K. Venayagamoorthy, "Recognition of facial expressions using Gabor wavelets and learning vector quantization," Engineering Applications of Artificial Intelligence 21.7, pp. 1056-1064, 2008.

[12] H. Khusnuliawati, A. Y. Wijaya and R. Soelaiman, IDENTIFIKASI PARAMETER OPTIMAL DARI METODE FUZZY C-MEANS DAN ESTIMASI ROBUST SPASIAL PADA SEGMENTASI CITRA DENGAN NOISE, Surabaya: Teknik Informatika ITS, 2014.

[13] M. Yakno, J. Saleh and B. Rosdi, "Low contrast hand vein image enhancement," In Signal and Image Processing Applications (ICSIPA), pp. 390-392, 2011.

[14] A. Frangi, W. Niessen, K. Vincken and M. Viergever, "Multiscale vessel enhancement filtering," Medical Image Computing and Computer-Assisted Interventation-MICCAI'98, pp. 130-137, 1998.

[15] C. L. L. Hendriks, Structure Characterization Using Mathematical Morphology, Cris Luengo, 2003.

[16] S. Haas, R. Donner, A. Burner, M. Holzer and G. Langs, "Superpixel-Based interest points for effective bags of visual words medical image retrieval," Medical Content-Based Retrieval for Clinical Decision Support, pp. 58-68, 2011.

[17] A. Kumar and Y. Zhou, "Human Identification Using Finger Images," vol. XXI, no. TRANSACTIONS ON IMAGE PROCESSING, 2012. 